\title{
X-ray/ץ-ray Correlation in Seyfert 2 Galaxy NGC 4945
}

\author{
Rafał Wojaczyński ${ }^{1, \text { a) }}$ and Andrzej Niedźwiecki ${ }^{1, b)}$ \\ ${ }^{1}$ Department of Astrophysics, University of Łódź, Pomorska 149/153, 90-236 Łódź, Poland \\ ${ }^{a)}$ Corresponding author: rafal.wojaczynski@wp.pl \\ b) niedzwiecki@uni.lodz.pl
}

\begin{abstract}
We report the correlation between the X-ray and $\gamma$-ray emission found in our analysis of the Fermi/LAT and Swift/BAT data from 8 years of observations of the nearby Seyfert 2 galaxy NGC 4945. Using the BAT light-curves we determined summed periods of low and high X-ray flux and we found that the average $\gamma$-ray spectrum is harder in the latter (higher $\mathrm{X}$-ray flux level), with the difference of the $\gamma$-ray spectral index of $\sim 0.4$. The correlation indicates that the $\gamma$-rays are produced in the active nucleus of this Seyfert galaxy rather than by cosmic rays interacting with the interstellar medium (as assumed in the alternative starburst model). We compare NGC 4945 with two other $\gamma$-ray loud galaxies showing both starburst and AGN activities, NGC 1068 and Circinus.
\end{abstract}

\section{INTRODUCTION}

NGC 4945 is one of the nearest AGNs ( $D=3.8 \mathrm{Mpc}$ ) with the black hole mass of $M=1.4 \times 10^{6} \mathrm{M}_{\odot}$ from megamaser measurements [1]. It is the brightest Seyfert 2 galaxy in the hard X-ray range. The nucleus of NGC 4945 radiates at a variable rate of $L_{\mathrm{Bo}} / L_{\mathrm{Edd}} \sim 0.1-0.5$ [2]. The absorbing column density $\left(\sim 4 \times 10^{24} \mathrm{~cm}^{-2}\right)$ and the observed high-energy variability identify it as a transmission-dominated AGN.

NGC 4945, with NGC 1068 and Circinus, are also the only radio-quiet Seyfert galaxies from which $\gamma$-ray signals have been measured by Fermi/LAT [3]. However, these three galaxies exhibit both AGN and starburst activities and the origin of $\gamma$-rays is unclear. [4] favor the starburst origin for NGC 4945 and NGC 1068 based on their approximate agreement with some multi-wavelength correlations found in other star-forming galaxies. However, Circinus does not follow these correlations and actually its $\gamma$-ray luminosity significantly exceeds the calorimetric limit (in which the total energy of cosmic rays is transformed into the production of $\gamma$-rays [5]). Furthermore, detailed star-burst models fail to describe NGC 1068 (e.g. [6], [7]).

On the other hand, the three $\gamma$-ray loud Seyfert galaxies show a striking similarity of their X-ray and $\gamma$-ray Eddington ratios ([8]; see Figure 4 below). This motivates us to consider the alternative model, with emission from the active nucleus, and we look for a correlation between the X-ray and $\gamma$-ray emission. The above noted large variations of the X-ray flux which may be directly probed, make NGC 4945 the best target for such a study. The $\mathrm{X}$-ray/Y-ray correlation cannot be studied in NGC 1068, which is fully reflection dominated and no X-ray variability is observed. Circinus, in turn, is strongly contaminated by the Galactic plane. 


\section{METHODS}

We use the Fermi/LAT (100 MeV - $100 \mathrm{GeV}$ ) and Swift/BAT ${ }^{1}(15-50 \mathrm{keV})$ data from observations performed by these detectors between 2008 August 4 and 2016 August 15. The hard X-ray flux from NGC 4945 varies on timescales of $\sim 10^{4} \mathrm{~s}$ ([2], [9]). However, it is approximately constant on time-scales sufficiently long to accumulate a statistically significant $\gamma$-ray signal. E.g. the yearly-averaged BAT fluxes deviate from the average value of $F_{\mathrm{X}}=$ $1.59 \times 10^{-3} \mathrm{ph} / \mathrm{cm}^{2} / \mathrm{s}$ by at most $15 \%$ (all the time-averaged values are computed following the method of [10]). The significance of the LAT signal for one-year intervals is only TS 50 .

Then, even if the $\gamma$-rays are correlated with X-rays in NGC 4945, we do not expect to reveal it in continuous data sets comprising observations carried out by LAT over sufficiently long periods for an adequate significance of the $\gamma$-ray signal. We, therefore, analyze intermittent data sets including days with different X-ray flux levels, as determined by the daily-averaged fluxes in the BAT light curve. All days with the available BAT count rate are split into two approximately equal sets, with the rate lower ( $\mathrm{L}$; 1393 days) and higher $\left(\mathrm{H} ; 1390\right.$ days) than $1.71 \times 10^{-3}$ $\mathrm{ph} / \mathrm{cm}^{2} / \mathrm{s}$. To test the effect of possible misclassification of the X-ray flux level due to short exposure time, we also define sets denoted by $\mathrm{L}_{5}$ and $\mathrm{H}_{5}$, using a similar procedure but only with the daily-average fluxes with the total exposure time of at least $5 \mathrm{ks}$. Here, with the median rate of $1.67 \times 10^{-3} \mathrm{ph} / \mathrm{cm}^{2} / \mathrm{s}$, we get 920 days in set $\mathrm{L}_{5}$ and 920 days in set $\mathrm{H}_{5}$. The average X-ray photon flux in $\mathrm{L} / \mathrm{L}_{5}$ is lower by a factor of $\sim 5$ than in $\mathrm{H} / \mathrm{H}_{5}$.

The LAT events are then summed over sets $\mathrm{L} / \mathrm{L}_{5}$ and $\mathrm{H} / \mathrm{H}_{5}$ and a standard Fermi analysis is applied to thus obtained sets of events. Specifically, we use Pass 8 data within radius $10^{\circ}$ centered at the position of NGC 4945, with standard templates for isotropic and galactic backgrounds; all 3FGL (Third LAT Catalogue [11]) sources within the radius of $15^{\circ}$ are taken into account.

\section{RESULTS}

Our results for the total Fermi data set (the top row in Table 1 and the top panel in Figure 2) are consistent with those reported for NGC 4945 in 3FGL. However, all our analyses of datasets selected based on the X-ray flux indicate that the spectrum of the $\gamma$-ray radiation differs between sets $L / L_{5}$ and $H / H_{5}$. The power law fits (Table 1) show that the spectrum is harder, with $\Delta \Gamma \simeq 0.4$, in set $\mathrm{H} / \mathrm{H}_{5}$. Consistently, the TS maps in Figure 1 show that in the 0.1-1 GeV and 3-100 GeV energy ranges the significance differs by $\sim 5 \sigma$ between set $\mathrm{L}$ and $\mathrm{H}$, with the $\gamma$-ray brightness reversal corresponding to the change of the X-ray flux level.

TABLE 1. Results of the power-law fits to NGC 4945 in the $100 \mathrm{MeV}-100 \mathrm{GeV}$ range; $\mathrm{F}_{\mathrm{X}}$ is the photon flux in the $15-50$ $\mathrm{keV}$ range in units of $10^{-3}$ photons $/ \mathrm{cm}^{2} / \mathrm{s}$; $\Gamma$ is the power-law photon index and $\mathrm{F}$ is the photon flux in the $0.1-100 \mathrm{GeV}$ range in units of $10^{-8}$ photons $/ \mathrm{cm}^{2} / \mathrm{s}$;

\begin{tabular}{lcccc}
\hline & $\mathbf{F}_{\mathbf{X}}$ & $\boldsymbol{\Gamma}$ & $\mathbf{F}$ & TS \\
\hline Total Fermi dataset (8 years) & $1.59 \pm 0.02$ & $2.34 \pm 0.04$ & $1.9 \pm 0.1$ & 390 \\
Low X-ray (L; 1393 days) & $0.54 \pm 0.03$ & $2.48 \pm 0.07$ & $2.7 \pm 0.3$ & 210 \\
High X-ray (H; 1390 days) & $2.87 \pm 0.04$ & $2.13 \pm 0.08$ & $1.2 \pm 0.2$ & 190 \\
Low X-ray (L5; 920 days) & $0.57 \pm 0.04$ & $2.46 \pm 0.09$ & $2.5 \pm 0.4$ & 136 \\
High X-ray (H; 920 days) & $2.75 \pm 0.04$ & $2.05 \pm 0.09$ & $1.1 \pm 0.2$ & 140 \\
\hline
\end{tabular}

The change of the slope of the $\gamma$-ray spectrum between low and high X-ray flux levels is also clearly seen in the spectral energy distributions (SED) shown in Figure 2 and we note that the results for sets $\mathrm{L}$ and $\mathrm{H}$ are consistent with these for sets $\mathrm{L}_{5}$ and $\mathrm{H}_{5}$.

\footnotetext{
${ }^{1}$ http://swift.gsfc.nasa.gov/docs/swift/results/transients
} 


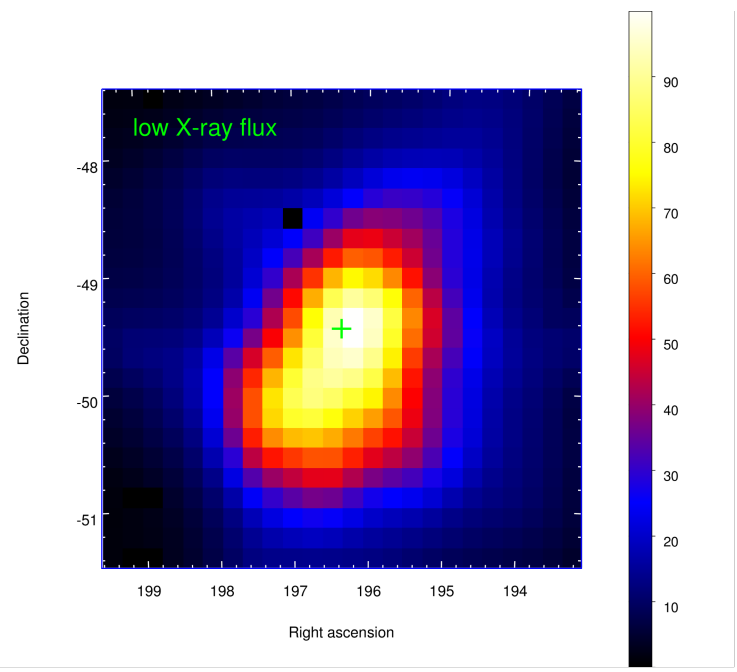

(a)

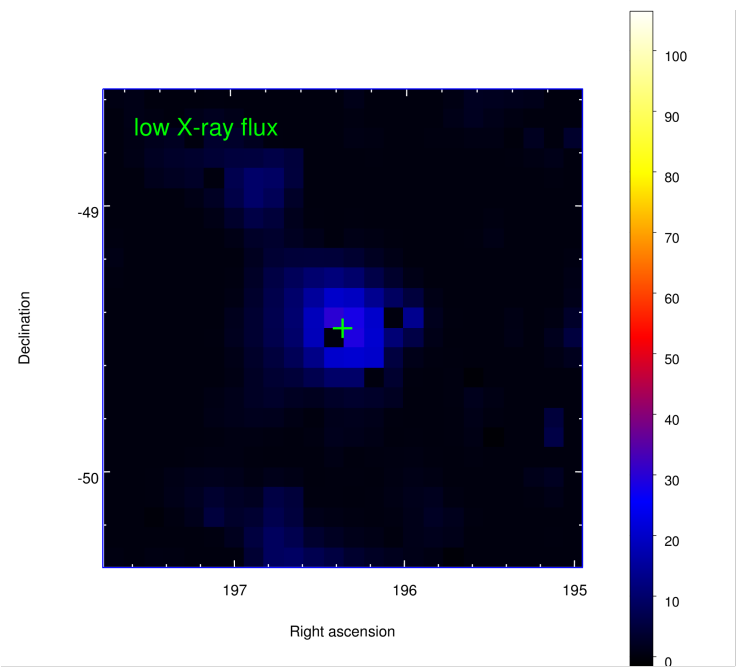

(c)

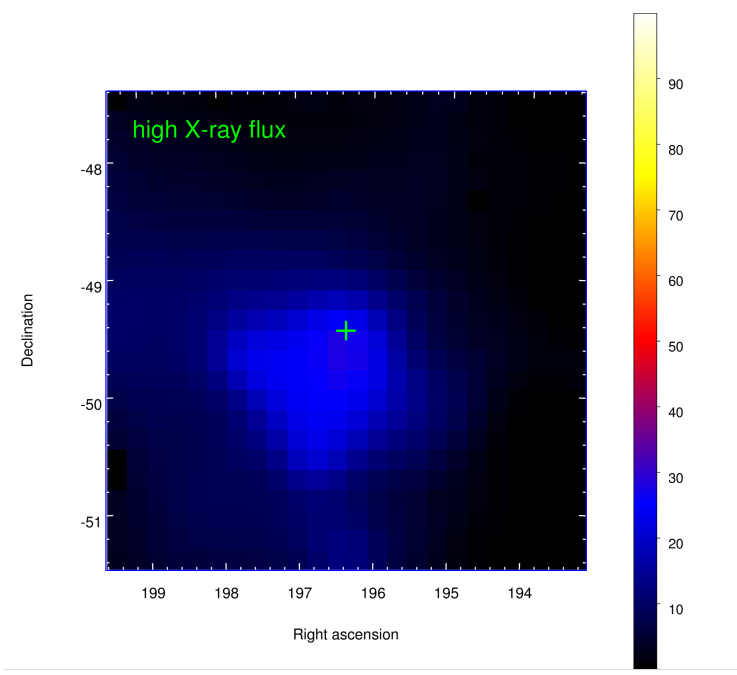

(b)

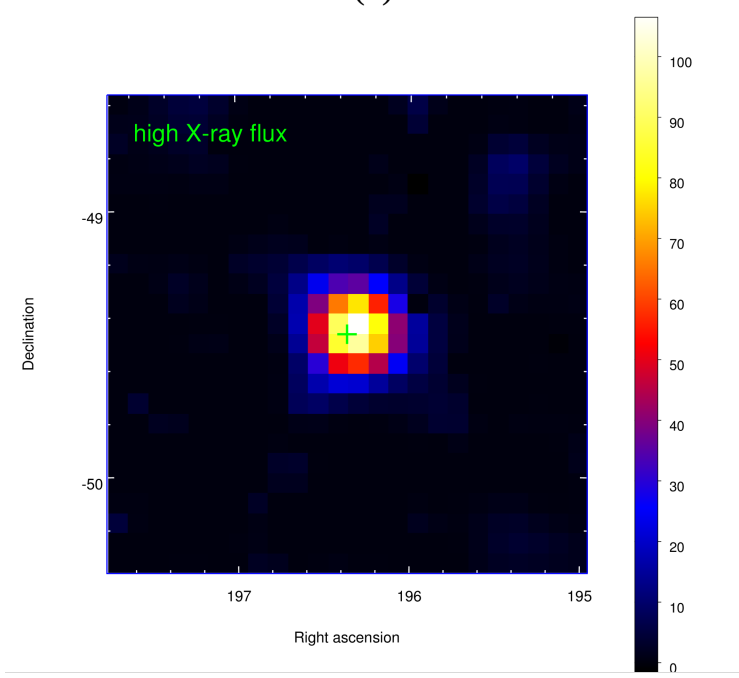

(d)

FIGURE 1. TS maps for the region around NGC 4945 built using the events detected during the low (L, left panels $a$ and $c$ ) and high (H, right panels $b$ and $d$ ) X-ray flux levels. In all panels the green cross shows the location of NGC 4945. In all panels, the 3FGL sources were subtracted from the maps except for NGC 4945. Top maps (a,b) are for events with $\mathrm{E}<1 \mathrm{GeV}$, bottom maps (c,d) are for events with $\mathrm{E}>3 \mathrm{GeV}$.

We also made a number of tests to estimate the magnitude of fluctuations that could affect our results. First, we analyzed the LAT datasets defined without considering the X-ray flux criterion and in all cases we found that they indicate a constant $\gamma$-ray signal. For example, the power-law fits to continuous 4-year intervals (Table 2) give parameters consistent with those of the total 8-year dataset within the uncertainty of the fitted values.

Secondly, we analyzed smaller data sets with LAT events summed over 400 or 800 days randomly drawn from either set L or set H. As we can see in Figure 3, all these shorter data sets consistently indicate the difference of the Y-ray spectrum between different X-ray flux levels. We compare these results with similar analyses of 400 or 800 days randomly drawn for NGC 253 and M 82, which are starburst galaxies and then their $\gamma$-ray signals should be constant in time. TS values for the power-law fits of these datasets in NGC 253 are similar to those in NGC 4945 with the same number of days; in M 82 the TS values are about twice higher. In both galaxies we note the spread of fitted parameters much smaller than that in NGC 4945. 
We conclude that difference of spectral parameters found for NGC 4945 in the data selected using the X-ray flux criterion is significantly larger than the statistical uncertainty of parameters typical for these TS values.

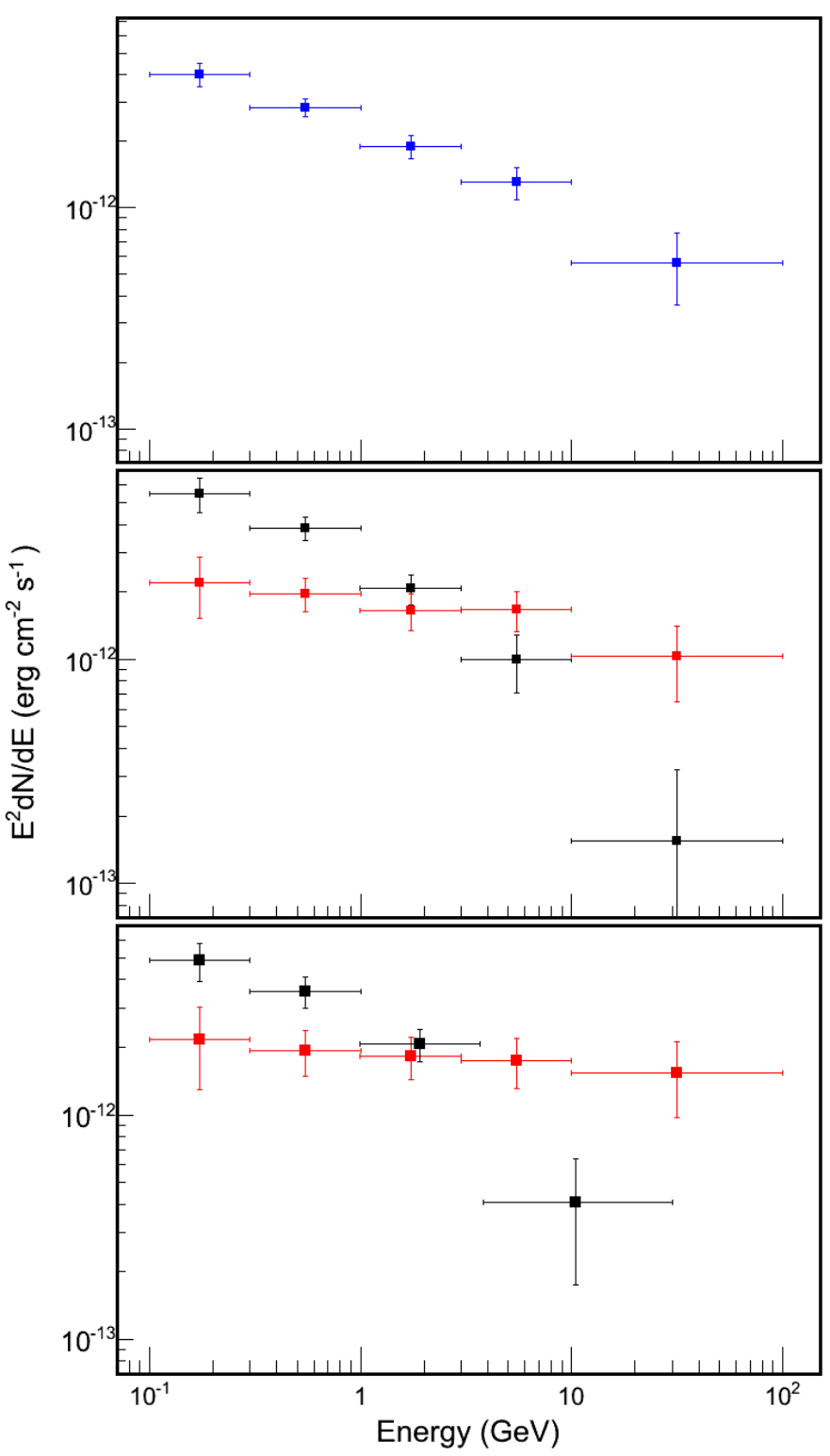

FIGURE 2. Spectral energy distributions (SED) for NGC 4945. The top panel is for the total (8 years) LAT dataset. The middle panel shows SED for set L (low X-ray flux; black) and H (high X-ray flux; red). The bottom panel shows SED for set $\mathrm{L}_{5}$ (black) and $\mathrm{H}_{5}$ (red). 
TABLE 2. Results of the power-law fits to NGC 4945 in the $100 \mathrm{MeV}-100 \mathrm{GeV}$ range for continuous 4-year intervals; $\Gamma$ is the power-law index and $F$ is the photon flux in the $0.1-100 \mathrm{GeV}$ range in units of $10^{-8}$ photons $/ \mathrm{cm}^{2} / \mathrm{s}$.

\begin{tabular}{lccc}
\hline & $\boldsymbol{\Gamma}$ & $\boldsymbol{F}$ & TS \\
\hline First 4 years & $2.30 \pm 0.08$ & $1.9 \pm 0.3$ & 200 \\
Second 4 years & $2.37 \pm 0.08$ & $2.0 \pm 0.3$ & 180
\end{tabular}

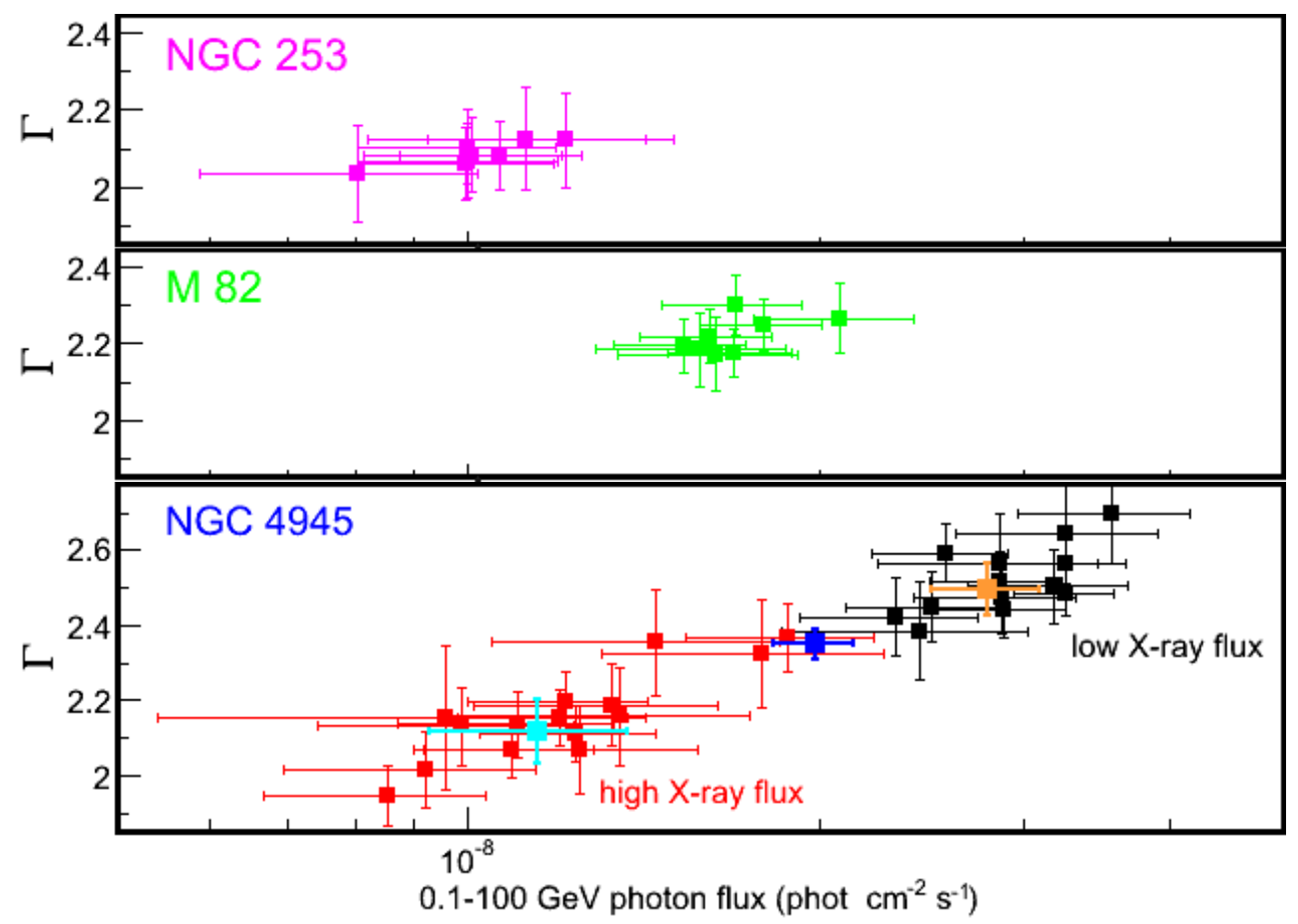

FIGURE 3. Parameters of the power-law fits in the $100 \mathrm{MeV}$ - $100 \mathrm{GeV}$ range for 400 or 800 days randomly drawn for NGC 253 (top) and M 82 (middle). In the bottom panel, the blue, orange and cyan point shows parameters of power-law fits to the total 8-year, L and H set in NGC 4945, respectively, as given in Table 1; the black points are for 400 or 800 days drawn randomly from set $\mathrm{L}$; the red points show the same for set $\mathrm{H}$.

\section{DISCUSSION}

In the starburst scenario, $\gamma$-rays are produced in the interstellar medium on large spatial scales, therefore, their flux and spectrum should be constant in time. Then, changes of the $\gamma$-ray spectrum, correlated with changes of the X-ray flux, revealed in our study indicate that in NGC 4945 the $\gamma$-ray emission is dominated by its active nucleus. Problems with applications of the starburst model to NGC 1068 and Circinus, noted above, and the similarity of $\mathrm{X}$-ray and $\mathrm{\gamma}$-ray Eddington ratios in all $\mathrm{\gamma}$-ray loud Seyferts (see Figure 4) suggest that the mechanism of $\gamma$-ray production in these objects is similar. 
The three $\gamma$-ray loud Seyfert galaxies are the most active AGNs in the local Universe, as indicated by their X-ray Eddington ratios. As discussed in [8], accretion flows in Seyfert galaxies may produce substantial $\gamma$-ray fluxes through inverse Compton scattering or $\pi^{0}$ production and decay. However, the observed $\gamma$-ray flux may be strongly attenuated by pair production in the X-ray radiation field. The strength of internal absorption depends on the geometry of the high-energy source. In AGNs with $L_{X} / L_{\mathrm{Edd}} \lesssim 10^{-3}$, the inner accretion flows are optically thin and their X-ray emission strongly attenuates the $\gamma$-ray radiation in the whole LAT energy range. At $L_{X} / L_{\mathrm{Edd}} \gtrsim 10^{-2}$, however, the hot flow is likely replaced by an optically thick disk. Such disks in AGNs radiate mostly in UV range and only photons with energies $\gtrsim 10 \mathrm{GeV}$ are absorbed in their radiation fields. Geometries of the high-energy source which are consistent with properties observed in NGC 4945 will be discussed in detail in our forthcoming paper (Wojaczyński et al., in preparation).

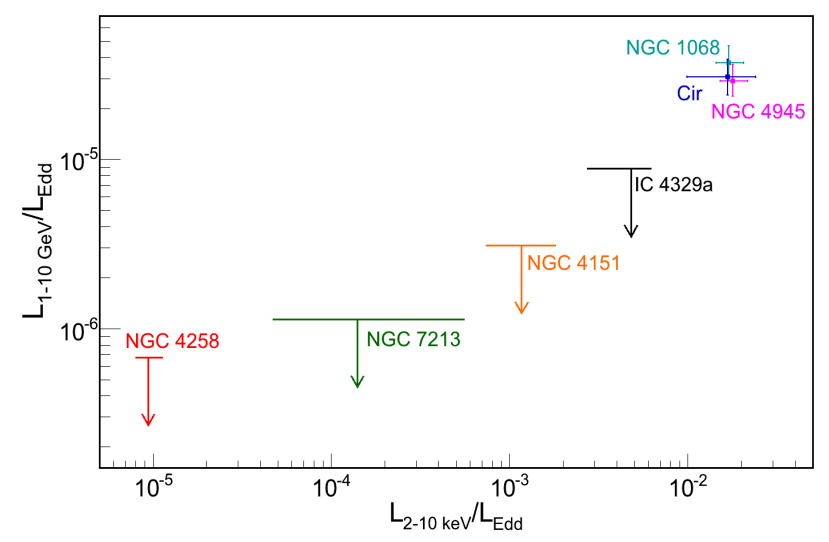

FIGURE 4. X-ray vs Y-ray Eddington ratios for nearby Seyfert galaxies ([8]).

\section{ACKNOWLEDGMENTS}

We thank Francesco Longo and Rachele Desiante for help with the Fermi/LAT data binned likelihood analysis. We made use of data and software provided by the Fermi Science Support Center, managed by the HEASARC at the Goddard Space Flight Center, and Swift/BAT transient monitor results provided by the Swift/BAT team. Rafał Wojaczyński has been supported by the Polish NCN grant UMO-2016/20/T/ST9/00386.

\section{REFERENCES}

1. Greenhill L.J., et al. 1997, ApJ, 481, 1.

2. $\quad$ Madejski G., et al. 2000, ApJ, 535, 2.

3. Ackermann M., et al. 2015, ApJ, 810, 14.

4. $\quad$ Ackermann M., et al. 2012 , ApJ, 755, 164.

5. Hayashida M., et al. 2013, ApJ, 779, 7.

6. Lenain J.-P., et al. 2010, A\&A, 524, 72.

7. Yoast-Hull T., et al. 2014, ApJ, 780, 137.

8. Wojaczynski R., et al. 2015, A\&A, 584, A20.

9. Puccetti S., et al. 2014, ApJ, 793, 1.

10. Ajello M., et al. 2008, ApJ, 673, 96.

11. Acero F., et al. 2015, ApJ, 218, 23. 